\title{
Evaluación formativa y compartida en la universidad: clarifi- cación de conceptos y propuestas de intervención desde la Red Interuniversitaria de Evaluación Formativa
}

\author{
Víctor Manuel López Pastor \\ E.U. Magisterio de Segovia, Universidad de Valladolid
}

RESUMEN: La finalidad de este trabajo es triple: (a)-realizar una introducción a la temática de la evaluación formativa y compartida en docencia universitaria y sus conexiones con el proceso de convergencia hacía el Espacio Europeo de Educación Superior (EEES); (b)-establecer una aclaración conceptual de los diferentes términos que se están utilizando actualmente en la literatura especializada en relación con la evaluación formativa y la participación del alumnado en la evaluación; (c)-realizar una breve introducción a la propuesta de Evaluación Formativa y Compartida en docencia universitaria que hemos desarrollado desde la Red. El contexto en el que se sitúa los trabajos de este monográfico es la Red de Evaluación Formativa y Compartida en docencia universitaria, su evolución y las diferentes líneas de innovación e investigación que está desarrollando desde hace seis años.

En el primer apartado realizamos una introducción al tema y su conexión con el proceso de Convergencia hacía el EEES. En el segundo llevamos a cabo una aclaración conceptual y terminológica sobre evaluación formativa y compartida en la universidad; también explicamos la confusión que ha surgido en los últimos años y apuntamos una solución para superarla. En el tercer apartado analizamos el estado de la cuestión sobre evaluación formativa y compartida en la universidad, así como las evidencias encontradas hasta el momento sobre sus ventajas respecto a los modelos tradicionales. En el cuarto y último apartado presentamos, de forma resumida, una propuesta concreta y contrastada de evaluación formativa y compartida en la universidad que puede ser aplicada a cualquier contexto.

Palabras clave: evaluación para el aprendizaje, evaluación alternativa, evaluación auténtica, evaluación orientada al aprendizaje, participación del alumnado en la evaluación.

Formative and shared assessment in higher education. Clarifying concepts and proposing interventions from the Formative and Shared Assessment Network

\footnotetext{
ABSTRACT: The purpose of this paper is threefold: (a)-making an introduction to the formative and shared assessment at Higher Education teaching and tracing its connections within the process of convergence towards the Higher Education European Area (HEEA), (b) - establishing a conceptual clarification of the different terms that are currently used in the literature in relation to the formative assessment and student participation in assessment, (c)- making a brief introduc- 
tion to the proposal of Formative and Shared Assessment in Higher Education that we have developed from our Network. The monograph included in this issue is based on the Formative and Shared Assessment Network in Higher Education , and it shows the evolution and the different lines of innovation and research that participants have been developed for six years.

In the first section of this paper an introduction of the Formative and Shared Assessment in Higher Education and its connection with the process of Convergence towards the HEEA is showed. In the second section we carry out a conceptual and terminological clarification on the Formative and Shared Assessment in Higher Education, suggesting some solutions for the terminological confusion on this issue that has arisen in recent years In the third section we analyze the state of the formative and shared assessment in Higher Education and the evidence found so far on its advantages regarding traditional models. In the fourth and final section we show a concrete proposal of formative and shared assessment in higher education that has been proved within our Network and which could be applied to other contexts.

Keywords: assessment for learning; alternative assessment, authentic assessment, learning-oriented assessment, student involvement in assessment

\section{INTRODUCCIÓN: LA EVALUACIÓN FORMATIVA Y COMPARTIDA EN LA DOCENCIA UNIVERSITARIA Y EL PROCESO DE CONVERGENCIA}

El proceso de Convergencia hacia el Espacio Europeo de Educación Superior (EEES) implica una serie de cambios en la forma de entender, organizar y llevar a cabo la docencia universitaria. El principal cambio es pasar de un paradigma centrado en la enseñanza del profesor, a un nuevo paradigma centrado en el aprendizaje del alumnado. Por ello, todas las asignaturas y titulaciones se organizan en "créditos ECTS", que miden las horas de estudio y trabajo del alumnado para generar los aprendizajes planificados, en vez de medir las horas de clase que imparte el profesor.

Existen otras clasificaciones dicotómicas que aportan interesantes matices a esta nueva forma de entender la enseñanza y el aprendizaje. La primera dicotomía que nos resulta muy significativa es la que diferencia entre el "Aprendizaje Bancario", que ha sido y sigue siendo el dominante y mayoritario en la enseñanza universitaria, y el "Aprendizaje Dialógico", que es el paradigma de aprendizaje que mejor permite el logro de los aprendizajes y las competencias complejas que requiere una formación universitaria. Freire $(1970,1990,1997)$ explica la diferencia entre los dos términos y sus implicaciones prácticas. Flecha (1998), Flecha y Puigvert $(1998,2005)$ profundizan en la temática del "aprendizaje dialógico", sus bases científicas y/o ejemplificaciones prácticas. La segunda dicotomía diferencia entre el "aprendizaje superficial" y el "aprendizaje profundo" en la enseñanza universitaria (Biggs, 1999), y defiende la idea de que la enseñanza universitaria debería potenciar el "aprendizaje profundo" en la mayor medida que sea posible. 
El cambio de paradigma afecta a todos los elementos curriculares. La evaluación y la metodología son los dos elementos que se ven más seriamente afectados y que más deberían evolucionar para ajustarse a esta forma de entender y desarrollar la docencia universitaria. En este sentido, un aspecto fundamental es la utilización de la evaluación como estrategia para mejorar y favorecer los aprendizajes, en vez de como simple certificación del éxito o fracaso en los mismos. La evaluación es un factor extraordinariamente influyente en los sistemas y procesos de trabajo y aprendizaje del alumnado, dado que condiciona la calidad de los mismos de una forma clara y directa (Biggs, 1999). Es importante ser consciente de que ninguna innovación curricular será efectiva si no va acompañada de innovaciones en el modo de concebir la evaluación (Bonsón y Benito, 2005).

El proceso de Convergencia hacia el EEES implica, al menos, los siguientes cambios en la forma de entender y practicar la evaluación en la docencia universitaria: (1)-Dar más importancia y dedicar más tiempo y esfuerzo a la evaluación continua y formativa que a la final y sumativa; (2)-Realizar una evaluación orientada a mejorar los aprendizajes y los procesos de enseñanza-aprendizaje y no sólo como control final de dichos procesos; (3)-Evaluar los diferentes tipos de aprendizaje y competencias que hemos planificado, en vez de sólo los evaluables con exámenes tradicionales; (4)-Evaluar también el proceso de aprendizaje y durante el mismo, no sólo el producto final.

Por otra parte, defendemos la importancia de hacer partícipe al alumno en la evaluación, tanto porque supone una mejora en sus aprendizajes y competencias, como porque ayuda a implicar al alumnado en su proceso de formación y su participación en la toma de decisiones. En este sentido, el avanzar hacia procesos de evaluación compartida ayuda a avanzar hacia modelos de educación para la producción de la sociedad, desde la aportación que sus miembros puedan hacer, más que una educación para la reproducción social. La implicación del alumnado en los procesos de evaluación de su aprendizaje debería ser una herramienta básica de trabajo cuando se pretende desarrollar la autonomía del alumnado y la capacidad de gestionar su propio aprendizaje. Para ello, uno de los instrumentos de trabajo más útiles son los procesos de evaluación formativa y compartida.

\section{DEFINICIONES Y ACLARACIONES CONCEPTUALES}

En este apartado vamos a explicar algunos conceptos sobre la temática de la evaluación formativa y compartida en docencia universitaria. También vamos a realizar algunas aclaraciones sobre posibles confusiones conceptuales que están surgiendo en los últimos años y realizamos algunas propuestas concretas para intentar solucionarlas.

En las dos últimas décadas, y sobre todo en los últimos años, están surgiendo diferentes términos estrechamente relacionados con el concepto de Evaluación Formativa: Evaluación Alternativa, Evaluación Auténtica, Evaluación para el Aprendizaje, Evaluación Formadora, Evaluación Orientada al Aprendizaje. Cada uno de estos términos surge con la intención de ofrecer matices nuevos 
a los términos ya existentes, o bien para desmarcarse de un tipo de prácticas evaluativas predominantes e intentar explicar una forma diferente de entender y llevar a cabo la evaluación en la práctica educativa. Desde nuestro punto de vista dichos términos aportan matices interesantes, aunque la mayor parte de sus planteamientos ya están recogidos en el concepto de "evaluación formativa", correctamente entendido tal concepto. En la tabla 1 presentamos una breve definición de cada uno de ellos.

Tabla 1. Conceptos Relacionados con la "Evaluación Formativa"

\section{Concepto en español e inglés o francés}

Evaluación Alternativa

(Alternative Assessment)

Evaluación Auténtica

(Autentic Assessment)

Evaluación para el

Aprendizaje

(Assessment for learning)

Evaluación Formadora

(Evaluation Formatrice)

Evaluación orientada al aprendizaje

(Learning-oriented

assessment)

Evaluación Formativa

(Formative Assessment)

\section{Definición}

Técnicas y métodos de evaluación que intentan superar a la metodología tradicional de evaluación, basada en la simple realización de pruebas y exámenes, con la finalidad única o principal de calificar.

Las técnicas, instrumentos y actividades de evaluación deben estar claramente aplicados en situaciones, actividades y contenidos reales de aprendizaje, o claramente relacionados con competencias profesionales. Por tanto, se opone a las situaciones puntuales y artificiales de evaluación, alejadas de la práctica real.

La evaluación debe estar claramente orientada a mejorar el aprendizaje del alumnado, en vez de estarlo al mero control de éste y su calificación. Algunos autores lo consideran como sinónimo del término "Evaluación formativa".

Busca promover la autorreflexión y el control sobre el propio aprendizaje. Se busca que el alumnado sea consciente de cómo aprende y qué tiene que hacer para seguir aprendiendo. Por tanto hace referencia al desarrollo de destrezas metacognitivas de carácter regulador.

Algunos autores anglosajones utilizan este concepto para hacer referencia a procesos similares de desarrollo de capacidades de autorregulación en el propio aprendizaje y de aprendizaje a lo largo de la vida. Para otros autores, también está fuertemente relacionado con la participación del alumnado en los procesos de evaluación y con el desarrollo de procesos y situaciones de evaluación auténtica.

Todo proceso de evaluación cuya finalidad principal es mejorar los procesos de enseñanza-aprendizaje que tienen lugar; está orientada a que el alumnado aprenda más y a que el profesorado aprenda a mejorar su práctica docente. 
En la Red de Evaluación Formativa y Compartida en Docencia Universitaria definimos "Evaluación Formativa" como: "todo proceso de constatación, valoración y toma de decisiones cuya finalidad es optimizar el proceso de enseñanzaaprendizaje que tiene lugar, desde un perspectiva humanizadora y no como mero fin calificador" (López Pastor et al, 2009, 2011).

Cuando se habla de la participación del alumnado en la evaluación suele ser conveniente realizar una serie de aclaraciones terminológicas de los conceptos más utilizados en la literatura especializada. En la tabla 2 presentamos una breve definición de cada uno de ellos. Para una mayor profundización nos remitimos a la obra colectiva López et al (2009).

Tabla 2. Conceptos Relacionados con la Participación del Alumnado en la Evaluación

\section{Concepto en español e inglés o francés}

"Autoevaluación"

(Self-Assessment)

"Coevaluación" o

"Evaluación entre iguales"

(Peer-assessment)

Evaluación Compartida (Shared Assessment; Coassessment; Collaborative assessment; Cooperative Assessment)

Autocalificación (Self-mark)

Calificación dialogada (Dialogic mark)

\section{Definición}

Suele referirse a la evaluación que una persona realiza sobre sí misma o sobre un proceso y/o resultado personal, aunque también puede ser grupal. Por tanto puede ser del alumnado o del profesorado.

Tradicionalmente en español se ha utilizado el concepto de "coevaluación" para referirse a la evaluación entre iguales. También puede ser individual (actividades de aprendizaje individuales) o colectiva (actividades en grupo). En estas últimas, suele ser conveniente valorar tanto los aspectos grupales como lo que cada individuo ha aportado al trabajo grupal (evaluación intragrupo).

Actualmente existe cierta confusión terminológica con la utilización de este término en español.

Procesos de diálogo que mantiene el profesor con su alumnado sobre la evaluación de los aprendizajes y los procesos de enseñanza-aprendizaje que tienen lugar. Este tipo de "diálogos" pueden ser individuales o grupales. Suelen estar basados o relacionados con procesos previos de autoevaluación y/o evaluación entre iguales. En inglés existen varios términos que podrían entenderse como sinónimos, aunque presentan matices diferentes $(\mathrm{Co}-$ assessment; Collaborative assessment; Cooperative Assessment).

Actualmente existe cierta confusión terminológica con la utilización de este término en español.

Proceso a través del cual cada alumno fija la calificación que cree merecer, normalmente tras un proceso previo en que el profesor acuerda con el alumnado los criterios de calificación.

Proceso por el cual alumnado y profesorado dialogan sobre la calificación definitiva y la acuerdan, en base a los criterios de calificación previamente establecidos. Puede ser individual, en pequeños grupos o en gran grupo. 
Actualmente en España parece existir cierta confusión con la utilización del concepto "coevaluación". Aunque tradicionalmente ha sido utilizado con el significado de "evaluación entre iguales", algunos docentes universitarios han realizado una traducción literal del término inglés "Co-assessment", utilizando el término "coevaluación" con el significado de "evaluación colaborativa". Esta situación está generando una confusión terminológica en los congresos y publicaciones especializadas, dado que un mismo término se está utilizando con dos significados diferentes. Una posible solución sería dejar de utilizar el término de "coevaluación" y utilizar los términos "evaluación entre iguales" o "evaluación colaborativa", en función de a qué tipo de evaluación nos estamos refiriendo realmente.

Por otra parte, nos gustaría realizar una última consideración sobre la participación del alumnado en la evaluación. Consideramos que se entre o no en procesos de Autocalificación y/o Calificación Dialogada, es importante tener claro que son procesos y momentos diferentes de la Evaluación Formativa y Compartida y que, a pesar de que en algún caso puedan estar relacionados, es conveniente dejar clara su separación, e incluso no entrar en este tipo de situación más veces de las estrictamente necesarias. Esto es, entendemos que los procesos de autocalificación y/o calificación dialogada sólo deberían realizarse al final de la asignatura, mientras que los procesos de evaluación formativa y compartida deberían llevarse a cabo durante todo el proceso de aprendizaje.

\section{LA EVALUACIÓN FORMATIVA Y COMPARTIDA EN LA DOCENCIA UNIVERSITARIA}

En la literatura especializada pueden encontrarse numerosos trabajos que defienden el desarrollo de sistemas de evaluación más formativos en docencia universitaria, por las numerosas ventajas que poseen en la mejora de la calidad de la enseñanza y los niveles de aprendizaje en la educación superior (Barberá, 2003; Biggs, 1999; Bonsón y Benito, 2005; Boud y Falchikow, 2007; Brown \& Glasner, 1999; Carless, Joughin \& Mok, 2006; Falchikov, 2005; Gibbs, 2003; Knight, 2005). En obras anteriores (López-Pastor et al, 2009) hemos recopilado algunas de estas ventajas: (1)-suele mejorar considerablemente la motivación e implicación del alumnado en el proceso de aprendizaje; (2)- Ayuda a corregir a tiempo las lagunas y problemáticas que surgen en el proceso de enseñanza-aprendizaje, por lo que permite mejorar los procesos de aprendizaje del alumnado, así como los procesos de enseñanza-aprendizaje que se desarrollan en la universidad; (3)-constituye una experiencia de aprendizaje en sí misma; (4)-es la forma de evaluación más lógica y coherente cuando la docencia se basa en sistemas de Aprendizaje Dialógico y/o en los modelos centrados en el aprendizaje del alumnado y en el desarrollo de competencias personales y profesionales; (5)-facilita el desarrollo de la capacidad de análisis crítico y la autocrítica; (6)-desarrolla la responsabilidad y autonomía del alumnado en los procesos de aprendizaje, con lo que adquiere un gran potencial de cara al desarrollo de estrategias de aprendizaje 
permanente (Lifelong Learning); (7)-suele mejorar el rendimiento académico, como efecto lógico de las ventajas enunciadas anteriormente.

Por otra parte, en la literatura especializada también pueden encontrarse numerosos trabajos, propuestas y experiencias sobre la participación del alumnado universitario en los procesos evaluativos: Biggs (1999), Brew (1995, 2003), Boud (1995), Boud y Falchikow (2007), Bretones (2002), Brown \& Dove (1990), Brown, Bull \& Pendlebury (1997), Brown y Glasner (1999); Brown y Knight (1994), Dochy, Segers y Sluijsmans (1999), Falchikov (2005), Fernández-Balboa (2005, 2006); Freire (1990), López, Fernández-Balboa, Santos y Fraile, (2011); López-Pastor et al, (2009), etc.

En los últimos 10 años se están acumulando numerosas evidencias de que es viable desarrollar asignaturas en la universidad española con sistemas de evaluación continua, formativa y compartida, claramente orientada al aprendizaje del alumnado (Busca, Pintor, Martínez y Peire, 2010; López-Pastor, 2009; LópezPastor, 2011; Martínez, Martín y Camplloch, 2010; Santos, Martínez y López, 2009; Vallés, Ureña y Ruiz, 2011; Zaragoza, Luis y Manrique, 2008). Este tipo de sistemas tiene una serie de ventajas, pero también algunos inconvenientes y problemáticas, que pueden irse solucionando progresivamente a través de espirales y ciclos de investigación-acción (Capllonch, Buscà, Martín, Martínez y Camerino, 2008; López, Martínez y Julián, 2007; López, Castejón, Sicilia, Navarro y Webb, 2011; Navarro, Santos, Busca, Martínez y Martínez, 2010; Pérez et al, 2008).

Los resultados de varias investigaciones también parecen indicar que este tipo de sistemas de evaluación favorecen la mejora de los resultados de aprendizaje del alumnado, así como las tasas de éxito y el rendimiento académico y que no suponen una sobrecarga de trabajo tan grande como se suele creer (Castejón, López, Julián y Zaragoza, 2011; Julián, Zaragoza, Castejón y López, 2010; López, Pintor, Muros y Webb, 2012).

\section{UNA PROPUESTA CONCRETA DE EVALUACIÓN FORMATIVA Y COM- PARTIDA EN LA UNIVERSIDAD}

Durante los últimos quince años hemos estado trabajando en una propuesta genérica para el diseño y desarrollo de sistemas de evaluación formativos y compartidos en docencia universitaria, en base a la experiencia que habíamos ido acumulando sobre el tema en la formación inicial del profesorado (López Pastor et al, 2009). A continuación vamos a desarrollar esquemáticamente dicha propuesta.

Los aspectos clave de la propuesta: la evaluación formativa y compartida y evolución de la Red de Evaluación Formativa en Docencia Universitaria

De las diferentes experiencias y procesos de evaluación que hemos generado en todos estos años, los dos aspectos que consideramos más importantes son: 
(a)-El esfuerzo por desarrollar una Evaluación eminentemente Formativa; (b)-El interés por desarrollar procesos e instrumentos que faciliten la realización de una Evaluación Compartida.

Como explicamos en el apartado anterior, ello implica poner el énfasis en utilizar la evaluación para mejorar los procesos de enseñanza-aprendizaje que tienen lugar, así como implicar al alumnado en el proceso de evaluación, de manera que la mayor parte del tiempo dedicado a la evaluación se convierta en un proceso de diálogo y toma de decisiones mutuas, orientadas a la mejora. En este sentido supone una clara alternativa a los modelos tradicionales de evaluación, únicamente dirigidos a la calificación del alumnado.

La realización de procesos de Evaluación Compartida y Formativa está fundamentada en la búsqueda de una coherencia entre nuestra práctica docente y los planteamientos epistemológicos y pedagógicos en que basamos nuestra teoría y práctica educativa. En este sentido hemos desarrollado una serie de instrumentos y sistemas de evaluación basados en la propuesta. Este sistema ha sido puesto en práctica, investigado, evaluado y mejorado durante los últimos doce años, demostrando su viabilidad y adecuación. Desde hace cinco años la Red Interuniversitaria de Evaluación Formativa y Compartida en Docencia Universitaria está generando una mayor difusión, experimentación, innovación e investigación sobre la temática, que nos está permitiendo profundizar, transferir y ampliar dichas propuestas en un marco contextual mucho más amplio. En dicha Red están implicadas actualmente alrededor de 65 personas de 21 universidades y 12 áreas de conocimiento diferentes (López et al, 2009, 2011; Navarro et al, 2010; Santos, Martínez y López, 2009). También se están generando diferentes estudios con los datos empíricos obtenidos en las numerosas experiencias llevadas a cabo (Busca, Pintor, Martínez y Peire, 2010; Capllonch et al, 2008; Castejón, López, Julián y Zaragoza, 2011; Julián, Zaragoza, Castejón y López, 2011; López, FernándezBalboa, Santos \& Fraile, 2011; López, Pintor, Muros \& Webb, 2012; Martínez, Martín y Camplloch, 2010; Valles, Ureña, Ruiz, 2011; Zaragoza, Luis y Manrique, 2008). En ellos se presentan los resultados obtenidos sobre viabilidad, ventajas, inconvenientes, soluciones, efectos en el rendimiento académico y efectos en la carga de trabajo para alumnado y profesorado.

\section{El desarrollo de la propuesta "Evaluación Formativa y Compartida en Edu- cación Superior"}

La propuesta se ha desarrollado a través de tres pasos sucesivos y encadenados. El primero fue elaborar unos Criterios de Calidad Evaluativa que debían cumplir un sistema de evaluación para poder considerarle "evaluación formativa y compartida". El segundo fue fijar algunas líneas de actuación (técnicas e instrumentos), de modo que cada docente pueda encontrar el sistema de evaluación adecuado para su caso. El tercero es ponerlo en práctica e iniciar ciclos de investigación-acción (plan, acción, observación, análisis) para irlos mejorando progresivamente, curso a curso. 


\section{Los Criterios de Calidad Educativa, entendidos como posibles Principios de Procedimiento}

Como intento de encontrar respuestas a estas preguntas elaboramos una serie de criterios de "Calidad Educativa" que deberíamos intentar cumplir en los sistemas e instrumentos de evaluación que diseñábamos y utilizábamos. En este sentido, funcionan como "Principios de Procedimiento", que nos orientan a la hora de decidir cómo poner en práctica los sistemas de evaluación concretos en cada contexto y asignatura. Son los siguientes: (1)-Adecuación respecto al diseño curricular; respecto a las características del alumnado y el contexto; y respecto a los planteamientos docentes; (2)-Relevancia, evaluar lo más importante, lo esencial; (3)-Veracidad, asegurar la credibilidad de la evaluación; (4)-Formativa, que sirva para mejorar el proceso y aprender más; (5)-Integrada en los procesos cotidianos de enseñanza-aprendizaje, respecto a los diferentes sujetos de la evaluación (profesorado, procesos, alumnado) y los diferentes ámbitos, contenidos y competencias a conseguir; (6)-Viable, en las condiciones reales de docencia; y (7)- Ética, tanto en el tratamiento de la información obtenida, como en el uso del poder que implica la calificación.

\section{Una propuesta basada en cinco líneas de actuación}

Proponemos trabajar en torno a cinco líneas que agrupan los diferentes tipos de instrumentos y dinámicas de evaluación, en las cuales venimos investigando durante los últimos años. Entendemos que estas líneas pueden y deben ser complementarias y, en ningún caso, excluyentes entre sí. Esto es, cada docente debe seleccionar las más adecuadas para su contexto y circunstancias, complementando entre dos o más de ellas. Son las siguientes: (a)-El cuaderno del profesor, desde los muy estructurados hasta los poco o nada estructurados; (b)-Actividades de aprendizaje específicas, como: prácticas de laboratorio, proyectos, estudios de caso, prácticas de campo, etc.; (c)-Las producciones del alumnado y las fichas de seguimiento del alumnado; (d)-Los procesos de autoevaluación, evaluación entre iguales y evaluación compartida; (e)-Dinámicas y ciclos de investigación y/o evaluación.

La puesta en práctica y los procesos de mejora a través de ciclos y espirales de investigación-acción

Nuestra propuesta entiende que cada contexto docente es único y que, por tanto, cada docente tiene que diseñar el sistema de evaluación formativa que mejor se ajuste a su asignatura y programa, a partir de los criterios y líneas de actuación recién explicadas. Posteriormente tiene que ponerlo en práctica e irlo mejorando progresivamente, a través de ciclos de investigación-acción (plan, recogida de datos, análisis y decisiones de mejora). El desarrollo de ciclos de investigación-acción encadenados permite ir mejorando y perfeccionando los 
sistemas de evaluación año a año. Si dichos ciclos se realizan de forma colectiva, junto a compañeros del mismo centro o centros cercanos, a través de proyectos de innovación docente, el proceso de mejora y aprendizaje suele ser mucho más rápido y eficaz.

Estas son las dinámicas básicas de la Red, en las que estamos implicados desde hace 6 cursos: (a)-diseño y desarrollo de sistemas de evaluación formativa y compartida en la universidad; (b)-su desarrollo práctico y su perfeccionamiento y mejora constante a través de ciclos de investigación-acción, tanto a nivel local como a nivel nacional. La realización de un congreso internacional anual sobre la temática nos permite reunirnos periódicamente para analizar los principales problemas que surgen y para buscar soluciones de mejora e ir perfeccionando progresivamente la calidad de nuestros sistemas de evaluación y de los procesos de aprendizaje de nuestro alumnado. Pueden encontrarse ejemplos concretos de experiencias de evaluación formativa y compartida en docencia universitaria en las actas de dichos congresos, así como en López et al, (2009) y Santos, Martínez y López, (2009).

La docencia universitaria es una profesión extraordinariamente compleja y diversa. Por ello es posible que no existan recetas mágicas que valgan para todas las asignaturas y contextos. Sin embargo quisiera destacar que existen ya propuestas claras, concretas y contrastadas, así como un considerable número de experiencias de innovación publicadas que pueden servir de orientación y ayuda a la hora de planificar el sistema de evaluación formativa que mejor se ajusta a nuestra propia práctica. Es importante aprender de las experiencias de innovación que han realizado otros compañeros, para poder mejorar la propia práctica. Confiamos en que este monográfico puede ser de alguna ayuda en ese sentido.

\section{AGRADECIMIENTOS}

Quiero reflejar mi profundo agradecimiento a Alvaro Sicilia, por las detalladas revisiones que ha hecho del manuscrito y por todas las sugerencias aportadas para mejorar la calidad del documento final.

\section{REFERENCIAS}

Barberá, E. (2003) Estado y tendencias de la evaluación en educación superior. Revista de la Red Estatal de Docencia Universitaria, 3(2), 47-60.

Biggs, J. B. (2005). Calidad del aprendizaje universitario. Madrid: Narcea.

Bonsón, M., y Benito, A. (2005). Evaluación y Aprendizaje. En A. Benito y A.

Cruz (coords.), Nuevas claves para la docencia universitaria en el Espacio Europeo de Educación Superior (pp. 87-100). Madrid: Narcea.

Boud, D. (1995). Enhancing Learning Through Self-Assessment. London: Routledge.

Boud, D., y Falchikov, N. (2007). Rethinking Assessment in Higher Education. Learning for the long term. Oxon: Routledge. 
Bretones Román, A. (2002). La participación del alumnado en la evaluación de sus aprendizajes. Revista Kikiriki-Cooperación Educativa, 65, 6-15.

Brew, A. (1995). Self-assessment in a variety of domains. Boud, D. (ed.) Enhancing Learning Through Self-Assessment. (pp 129-154). London: Kogan Page.

Brew, A. (2003). La autoevaluación y la evaluación por los compañeros. Brown, S. y Glasner, A. Evaluar en la universidad. Problemas y nuevos enfoques. (pp 179-190). Madrid: Narcea.

Brown, S., y Dove, P. (Eds). (1990). Self and peer assessment. SEDA Paper $n^{\circ}$ 72. Birmingham: SEDA. 1990.

Brown, S., y Glasner, A. (Ed.). (2003). Evaluar en la universidad. Problemas y nuevos enfoques. Madrid. Narcea.

Brown, G., Bull, J., y Pendlebury, M. (1997). Assessing Student Learning in Higher Education. London: Routledge.

Brown, S., y Knight, P. (1994). Assessing Learners in Higher Education. London: Kogan Page.

Busca, F., Pintor, P., Martínez, L., y Peire, T. (2010). Sistemas y procedimientos de Evaluación Formativa en docencia universitaria. Estudios sobre Educación, 18, 255-276.

Castejón Oliva, F. J. y López Pastor, V. M., Julián Clemente, J., y Zaragoza Casterad, J. (2011). Evaluación formativa y rendimiento académico en la Formación inicial del profesorado de Educación Física. Revista Internacional de Medicina y Ciencias de la Actividad Física y el Deporte, 42, 328-346. Obtenido de: http://cdeporte.rediris.es/revista/revista42/artevaluacion163.htm

Capllonch, M., Buscà, F., Martín, M., Martínez, L., y Camerino, O. (2008). Trabajo docente en equipo en evaluación formativa. Redes de trabajo y trabajo en red en Educación Física. Revista Fuentes, 8, 219-234. Obtenido de: http:// www.revistafuentes.org

Carless, D., Joughin, G., y Mok, M. M. C. (2006). Learning-oriented Assessment: Principles and Practice. Assessment \& Evaluation in Higher Education, 31, 4, 395-398.

Dochy, F., Segers, M., y Sluijsmans, D. (1999). The Use of Self-, Peer and Co-assessment in Higher Education: a review. Studies in Higher Education, 24(3), 331-350.

Falchikov, N. (2005). Improving Assessment Through Student Involvement. Practical solutions for aiding learning in higher and further education. Oxon: Routledge.

Fernández-Balboa, J. M. (2005) La autoevaluación como práctica promotora de la democracia y la dignidad. En A. Sicilia y J. M. Fernández-Balboa (Coord.), La otra cara de la educación física: la educación física desde una perspectiva crítica. Barcelona: Inde.

Fernández-Balboa, J. M. (2006) Dignity and democracy in the college classroom: The practice of self-evaluation. In Rebecca A. Goldstein Useful Theory: Making Critial Eduation Practical. New York: Peter Lang Publishing. 
Flecha, R. (1998). Compartiendo palabras. Barcelona. Paidós.

Flecha, R., y Puigvert, L. (1998). Aportaciones de Paulo Freire a la educación y las ciencias sociales. Revista Interuniversitaria de Formación del Profesorado, 33, 21-28.

Flecha, R., y Puigvert, L. (2005) Formación del profesorado en las comunidades de aprendizaje. Revista Colombiana de Educación, 48, 13-36.

Freire, P. (1970). La educación como práctica de libertad. México: Siglo XXI.

Freire, P. (1990). La naturaleza política de la Educación: Cultura, Poder y Liberación”. Barcelona: Paidos-MEC.

Freire, P. (1997). A la sombra de este árbol. Barcelona: El Roure.

Gibbs, G. (2003). Uso estratégico de la evaluación en el aprendizaje. En S. Brown y A. Glasner (Ed.), Evaluar en la universidad. Problemas y nuevos enfoques (61-75). Madrid: Narcea.

Julián, J., Zaragoza, J., Castejón, F.J., y López-Pastor, V.M. (2010). Carga de trabajo en diferentes asignaturas que experimentan el sistema ECTS. Revista Internacional de Medicina y Ciencias de la Actividad Física y el Deporte, 10, 38, 218-233. Obtenido de Http://cdeporte.rediris.es/revista/revista38/artcarga151.htm.

Knight, P. (2005) El profesorado de Educación Superior. Madrid: Narcea.

López-Pastor, V. M. (Coord.) (2009). La Evaluación Formativa y Compartida en Docencia Universitaria: propuestas, técnicas, instrumentos y experiencias. Madrid: Narcea.

López-Pastor, V. M., Martínez-Muñoz, L. F., y Julián, J. A. (2007) La Red de Evaluación Formativa, Docencia Universitaria y Espacio Europeo de Educación Superior (EEES). Presentación del proyecto, grado de desarrollo y primeros resultados. Red-U. Revista de Docencia Universitaria, 2. Obtenido de http://www.redu.um.es/red_U/2

López-Pastor, V. M., Castejón, J., Sicilia-Camacho, A., Navarro, V., y Webb, G. (2011). The process of creating a cross-university network for formative and shared assessment in higher education in Spain and its potential applications. Innovations in Education and Teaching International, 48(1), 79-90.

López-Pastor, V. M., Fernández-Balboa, J. M., Santos, M. L., y Fraile, A. (2011) Students' self-grading, professor's grading and negotiated final grading at three university programmes: analysis of reliability and grade difference ranges and tendencies. Assessment \& Evaluation in Higher Education, (first published on 21-april-2011 (iFirst).

López-Pastor, V. M. (2011). Good practice on Superior Education Evaluation: a Case of Formative and Sharing Evaluation. Journal of Tecnology and Science Education, 1(2), 1-12.

López-Pastor, V. M., Pintor, P., Muros, B., y Webb, G. (2012). Student performance and student and tutor workload in pilot projects relating to formative assessment strategies. Journal of Futher and Higher Education, 1-18. iFirst Article. Obtenido de: http://dx.doi.org/10.1080/0309877X.2011.644780

Martínez, L., Martín, M., y Camplloch, M. (2010). Una experiencia de desarro- 
llo profesional del docente universitario de Educación Física a través de una práctica crítica, reflexiva y colaborativa. Cultura y Educación, 21(1), 95-106.

Navarro, V., Santos, M., Busca, F., Martínez, L., y Martínez, L. F. (2010). La experiencia de la red universitaria española de evaluación formativa y compartida: proceso y abordaje. Revista Iberoamericana de Educación, 52(7), 1-13.

Pérez, A, Tabernero, B., López, V. M., Ureña, N., Ruiz, E., Capllonch, M., González, N., y Castejón, J. (2008). Evaluación formativa y compartida en la docencia universitaria: La concreción de cuestiones/clave para su aplicación en el camino hacia el Espacio Europeo de Educación Superior (EEES). Revista de Educación, 347, 435-451. Obtenido de: www.revistaeducacion.mepsyd. es. (consultado el 15/10/2008).

Santos, M., Martínez-Muñoz, L. F., y López-Pastor, V. M. (Coords.) (2009). La Innovación docente en el EEES. Almería: Universidad de Almería.

Valles, C., Ureña, N., y Ruiz, E. (2011). La Evaluación Formativa en Docencia Universitaria. Resultados globales de 41 estudios de caso. REDU. Revista de Docencia Universitaria, 9(1), 135-158. Obtenido de http://redaberta.usc.es/ redu/index.php/REDU

Zaragoza, J., Luis, J. C., y Manrique, J. C. (2008). Experiencias de innovación en docencia universitaria: resultados de la aplicación de sistemas de evaluación formativa. REDU. Revista de Docencia Universitaria, 4, 1-33. Obtenido de: http://www.redu.um.es/red_U/4. (consultado 20/06/2009). 
